\title{
An Output Encoding Problem and a Solution Technique
}

\author{
Subhasish Mitra, LaNae J. Avra and Edward J. McCluskey \\ Center for Reliable Computing \\ Departments of Electrical Engineering and Computer Science \\ Stanford University, Stanford, California 94305
}

\begin{abstract}
We present a new output encoding problem as follows: Given a specification table, such as a truth table or a finite state machine state table, where some of the outputs are specified in terms of 1 's, $O$ 's and don't cares, and others are specified symbolically, and assuming that the minimum number of bits are used to encode the symbolic outputs ( $\log _{2} n 7$ bits for $n$ symbolic outputs), determine a binary code for each symbol of the symbolically specified output column such that the total number of output functions to be implemented after encoding the symbolic outputs and compacting the columns is minimum. There are several applications of this output encoding problem, one of which is to reduce the area overhead while implementing scan or pseudo-random BIST in a circuit with one-hot signals. We develop an exact algorithm to solve the above problem and present experimental data to validate the claim that our encoding strategy helps to reduce the area of a synthesized circuit.
\end{abstract}

\section{INTRODUCTION}

Column compaction plays a major role in the synthesis of digital systems [1]. In column compaction, the number of output functions for a given specification is reduced by merging outputs which are logically equivalent, or can be made equivalent through assignment of don't cares. Two examples of output column compaction are shown in Fig. 1. Given a set of output columns, the problem of finding the smallest set that can be obtained by compacting the given set is related to the maximum clique partitioning problem, which is an NP-Complete problem [2]. This smallest set of compacted outputs is called the minimum cardinality output column cover. Column compaction can greatly reduce circuit area; this is not only true for the PLA implementation of the circuit [3] but also for multi-level logic circuits.

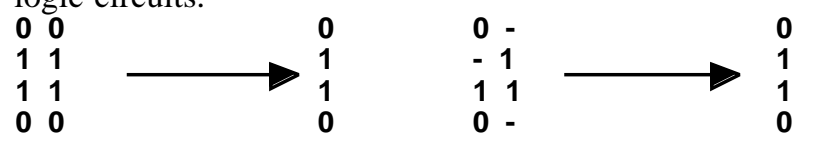

Figure 1. Examples of column compaction.

The different types of encoding problems, studied in the past, are the finite state machine (FSM) state encoding problem and the input and the output encoding problem. Techniques for FSM state encoding have been discussed in $[4,5,6,7,8,9,10]$. The input encoding problem has been reported in $[11,12]$. One version of the output encoding problem, with a goal to reduce the number of product terms of a logic function, has been discussed in [13]. Another version of the output encoding problem is discussed in [14]. A heuristic solution to the output encoding problem which uses column compaction to reduce the number of outputs after the symbols are encoded is given in [15]. However, this algorithm does not consider any existing non-symbolic (binary) outputs when determining the encoding for the symbols of the symbolic output column.

In this paper, we present an output encoding problem whose objective is different from those mentioned above. The input to our problem is a specification table of a combinational (or sequential) circuit in terms of a truth table (or state table) such that some of the outputs are specified in terms of 0's, 1's and don't cares while the other outputs are symbolically specified. The problem is to encode the symbols in the symbolic output column, using $\left\lceil\log _{2} n\right\rceil$ bits for $n$ symbolic outputs, so that after encoding, the cardinality of the output column cover computed using column compaction is minimum. Section 2 explains the motivation behind studying this type of an output encoding problem. Section 3 presents our output encoding algorithm for the case in which the specification table does not contain any don't cares in its output part. In Sec. 4, we extend the algorithm of Sec. 3 to handle don't cares in the outputs of the specification. Experimental results are reported in Sec. 5 followed by conclusion in Sec. 6.

\section{2 . MOTIVATION}

Consider the specification shown in Table 1. Output columns $c_{1}, c_{2}, c_{3}$ and $c_{4}$ of Table 1 are specified in terms of 1's, 0's and don't cares - they constitute the B-set, the bound set. The last output column of Table 1 is symbolic - it is referred to as the S-column, the symbolic column. In this paper, we will consider specification tables containing a single symbolic output column (S-column) for simplicity. We can handle cases with multiple symbolic output columns by choosing an appropriate ordering of the symbolic outputs and repeatedly applying the algorithm reported in this paper.

For Table 1, since we have seven distinct symbols in the S-column, we need 3 bits to encode them. Table 2(a) shows one possible encoding of the symbols in the Scolumn and Table 2(b) shows the truth table for the corresponding function to be realized after column compaction. In Table 2(b), c5, c6 and $\mathrm{c}_{6}$ represent the symbolic outputs. 
Table 1 . Truth table with symbolic output.

\begin{tabular}{|c|c|c|}
\hline Input & $\begin{array}{c}\text { Encoded Output } \\
\mathrm{c}_{1} \mathrm{c}_{2} \mathrm{c}_{3} \mathrm{c}_{4}\end{array}$ & $\begin{array}{c}\text { Symbolic } \\
\text { output }\end{array}$ \\
\hline 10101 & $\begin{array}{lllll}1 & 0 & 1 & 0\end{array}$ & $X_{1}$ \\
\hline 01100 & $\begin{array}{llll}0 & 0 & 1 & 0\end{array}$ & $X_{2}$ \\
\hline 10001 & $\begin{array}{llll}1 & 0 & 0 & 1\end{array}$ & $\mathrm{X}_{3}$ \\
\hline 01111 & $-1-0$ & $\mathrm{X}_{2}$ \\
\hline 11110 & $0-0-$ & $\mathrm{X}_{4}$ \\
\hline 01010 & $11-0$ & $\mathrm{X}_{5}$ \\
\hline 11111 & $10-0$ & $X_{1}$ \\
\hline $1110-$ & $0-11$ & $x_{6}$ \\
\hline 11011 & $\begin{array}{llll}1 & 0 & 1 & 1\end{array}$ & $X_{7}$ \\
\hline 01001 & $\begin{array}{llll}0 & 1 & 0 & 1 \\
\end{array}$ & $X_{4}$ \\
\hline
\end{tabular}

Table 2 ( a ). Encoding. Table 2 ( b ). Compacted Truth Table.

\begin{tabular}{|c|c|}
\hline Signals & Encoding \\
\hline $\mathrm{X}_{1}$ & 100 \\
$\mathrm{X}_{2}$ & 111 \\
$\mathrm{X}_{3}$ & 101 \\
$\mathrm{X}_{4}$ & 011 \\
$\mathrm{X}_{5}$ & 010 \\
$\mathrm{X}_{6}$ & 110 \\
$\mathrm{X}_{7}$ & 001 \\
\hline
\end{tabular}

\begin{tabular}{|c|cccc|ccc|}
\hline Input & \multicolumn{6}{|c|}{ Outputs } \\
& $\mathrm{c}_{1}$ & $\mathrm{c}_{2}$ & $\mathrm{c}_{3}$ & $\mathrm{c}_{4}$ & $\mathrm{c}_{5}$ & $\mathrm{c}_{6}$ & $\mathrm{c}_{7}$ \\
\hline 10101 & 1 & 0 & 1 & 0 & 1 & 0 & 0 \\
01100 & 0 & 0 & 1 & 0 & 1 & 1 & 1 \\
10001 & 1 & 0 & 0 & 1 & 1 & 0 & 1 \\
01111 & - & 1 & - & 0 & 1 & 1 & 1 \\
11110 & 0 & - & 0 & - & 0 & 1 & 1 \\
01010 & 1 & 1 & - & 0 & 0 & 1 & 0 \\
11111 & 1 & 0 & - & 0 & 1 & 0 & 0 \\
$1110-$ & 0 & - & 1 & 1 & 1 & 1 & 0 \\
11011 & 1 & 0 & 1 & 1 & 0 & 0 & 1 \\
01001 & 0 & 1 & 0 & 1 & 0 & 1 & 1 \\
\hline
\end{tabular}

Table 3 (a). Encoding.

\begin{tabular}{|c|c|}
\hline Signals & Encoding \\
\hline $\mathrm{X}_{1}$ & 110 \\
$\mathrm{X}_{2}$ & 010 \\
$\mathrm{X}_{3}$ & 101 \\
$\mathrm{X}_{4}$ & 001 \\
$\mathrm{X}_{5}$ & 100 \\
$\mathrm{X}_{6}$ & 011 \\
$\mathrm{X}_{7}$ & 111 \\
\hline
\end{tabular}

3 (b) . Compacted Truth Table.

\begin{tabular}{|c|c|}
\hline Input & $\begin{array}{c}\text { Output } \\
\underline{c_{1}} \underline{\mathrm{c}} 2 \mathrm{c}_{3} \underline{\mathrm{c}} \underline{4}\end{array}$ \\
\hline 10101 & $\begin{array}{llll}1 & 0 & 1 & 0\end{array}$ \\
\hline 01100 & $\begin{array}{llll}0 & 0 & 1 & 0\end{array}$ \\
\hline 10001 & $\begin{array}{llll}1 & 0 & 0 & 1\end{array}$ \\
\hline 01111 & $\begin{array}{llll}0 & 1 & 1 & 0\end{array}$ \\
\hline 11110 & $0-0-1$ \\
\hline 01010 & $\begin{array}{llll}1 & 1 & 0 & 0\end{array}$ \\
\hline 11111 & $\begin{array}{llll}1 & 0 & 1 & 0\end{array}$ \\
\hline $1110-$ & $0-11$ \\
\hline 11011 & $\begin{array}{llll}1 & 0 & 1 & 1\end{array}$ \\
\hline 01001 & $\begin{array}{llll}0 & 1 & 0 & 1\end{array}$ \\
\hline
\end{tabular}

It is possible to reduce the number of output columns after column compaction if we encode the symbols of Table 1 as shown in Table 3(a). The column compacted truth table is shown in Table 3(b). In Table 3(b), columns $\mathrm{c}_{1}, \mathrm{c}_{2}$ and $\mathrm{c}_{4}$ represent the symbolic outputs.

This output encoding problem has many applications. Digital systems are often specified in terms of a combination of binary valued and symbolic signals. This problem is applicable to reduce the area of scan-based [16] designs containing one-out-of-n (one-hot) signals, as described in [17]. This algorithm can serve as a preprocessing step for FSM state encoding.

\section{ENCODING ALGORITHM FOR FULLY SPECIFIED OUTPUTS}

In this section, we present our output encoding algorithm with the assumption that the output columns belonging to the B-set are fully specified with 1's and 0's for simplicity. We consider the example of Table 4 for illustration. We describe the three basic steps of our algorithm as follows:
Step 1 : Find Inconsistent Columns: Find all pairs of rows, $p$ and $q$, in Table 4 for which the S-column has the same value and $c_{i} \in \mathrm{B}$-set has different values. Then $c_{i}$ is said to be inconsistent. For this example, the B-set is $\left\{c_{1}, c_{2}, c_{3}, c_{4}, c_{5}, c_{6}\right\}$. Here, $c_{1}$ has a 1 in the second row and a 0 in the 4 th row; but the $S$-column has $X_{2}$ in both of these rows. Hence, $c_{1}$ is inconsistent. Column $c_{3}$ is also inconsistent for symbolic output $\mathrm{X}_{3}$.

Step 2: Create reduced consistent output table: Remove inconsistent columns from the specification table to obtain the consistent output table (COT). Merge equal rows of COT to obtain reduced consistent output table (RCOT). Table 5 is the RCOT for Table 4. Since there are 6 symbolic outputs, we use $m=3$ bits to encode them.

Step 3: $i$-column counting check: Any set of $i$ columns of the RCOT $(i \leq m)$ having any of the $2^{i}$ possible binary values of length $i$ appearing more than $2^{m-i}$ times in the rows of the RCOT cannot reduce the size of the output column cover after encoding the symbols. All sets of $i$ columns that satisfy this $i$-column counting check form elements of column-count-set- $i$ (CCS- $i)$. For Table 5, CCS-1 $=\left\{\left\{c_{2}\right\},\left\{c_{5}\right\},\left\{c_{6}\right\}\right\}, \operatorname{CCS}-2=\left\{\left\{c_{2}, c_{5}\right\}\right.$, $\left.\left\{c_{5}, c_{6}\right\},\left\{c_{2}, c_{6}\right\}\right\}, C C S-3=$ NULL. $\left\{c_{4}\right\}$ is not included in CCS-1 because the number of 1's in the c4 column is 5 (greater than 4). We terminate this step when either a CCS set is NULL or CCS- $m$ has been generated.

Table 4 . Specification Table.

\begin{tabular}{|cccccc|c|}
\hline $\mathrm{c}_{1}$ & $\mathrm{c}_{2}$ & $\mathrm{c}_{3}$ & $\mathrm{c}_{4}$ & $\mathrm{c}_{5}$ & $\mathrm{c}_{6}$ & Symbol \\
\hline 0 & 0 & 1 & 1 & 1 & 0 & $\mathrm{X}_{1}$ \\
$\mathbf{1}$ & 0 & 0 & 1 & 0 & 1 & $\mathbf{X}_{2}$ \\
1 & 0 & $\mathbf{0}$ & 1 & 1 & 1 & $\mathbf{X}_{3}$ \\
$\mathbf{0}$ & 0 & 0 & 1 & 0 & 1 & $\mathbf{X}_{2}$ \\
0 & 1 & 1 & 1 & 0 & 0 & $\mathrm{X}_{4}$ \\
0 & 1 & 0 & 1 & 1 & 0 & $\mathrm{X}_{5}$ \\
1 & 0 & 1 & 1 & 1 & 1 & $\mathrm{X}_{3}$ \\
1 & 1 & 0 & 0 & 0 & 0 & $\mathrm{X}_{6}$ \\
1 & 0 & $\mathbf{1}$ & 1 & 1 & 1 & $\mathbf{X}_{3}$ \\
1 & 0 & 0 & 1 & 0 & 1 & $\mathrm{X}_{2}$ \\
\hline
\end{tabular}

Table 5 . The RCOT.

\begin{tabular}{|c|c|}
\hline$c_{2} c_{4} c_{5} c_{6}$ & Symbol \\
\hline $\begin{array}{llll}0 & 1 & 1 & 0\end{array}$ & $\mathrm{X}_{1}$ \\
\hline $\begin{array}{llll}0 & 1 & 0 & 1\end{array}$ & $\mathrm{X}_{2}$ \\
\hline $\begin{array}{llll}0 & 1 & 1 & 1\end{array}$ & $X_{3}$ \\
\hline $\begin{array}{llll}1 & 1 & 0 & 0\end{array}$ & $X_{4}$ \\
\hline $\begin{array}{llll}1 & 1 & 1 & 0\end{array}$ & $\mathrm{X}_{5}$ \\
\hline $\begin{array}{llll}1 & 0 & 0 & 0\end{array}$ & $x_{6}$ \\
\hline
\end{tabular}

For Table 4, we can encode the symbols using three bits in such a way that two of the three columns, representing the symbolic outputs, can be merged with the existing output columns by column compaction. We choose any member of CCS-2, say, $\left\{c_{2}, c_{5}\right\}$. The first two bits in the encoding of a particular symbol will have the same pattern as the one present under the columns $c_{2}$ and $c_{5}$ in the row corresponding to that symbol in the RCOT. We determine the third bit such that the codes assigned to the symbols are distinct. Thus, referring to Table $4, \mathrm{X}_{1}$ can be encoded as $010, \mathrm{X}_{2}$ as $000, \mathrm{X}_{3}$ as $011, \mathrm{X}_{4}$ as $100, \mathrm{X}_{5}$ as 110 and $\mathrm{X}_{6}$ as 101. It is straightforward to prove the optimality of our solution [18]. It should be noted that if we allowed 4 bits to encode the symbolic outputs of Table 4, then all output columns corresponding to the four encoding bits could be merged with $c_{2}, c_{4}, c_{5}$ and $c_{6}$. In that case, we can iterate step 3 incrementing the number of encoding bits until all the encoding bit outputs can be merged with the 
existing outputs or we reach a point where we have tried to encode $n$ symbolic outputs using $n$ bits.

\section{ENCODING ALGORITHM FOR INCOMPLETELY SPECIFIED OUTPUTS}

The basic steps of the algorithm described in Sec. 3 can be extended to handle don't cares in the B-set.

Table 6 . Specification Table.

\begin{tabular}{|cccccc|c|}
\hline$c_{1}$ & $c_{2}$ & $c_{3}$ & $c_{4}$ & $c_{5}$ & $c_{6}$ & Symbolic \\
\hline 0 & - & 1 & 0 & - & 0 & $X_{1}$ \\
$\mathbf{1}$ & 0 & - & - & - & - & $\mathbf{X}_{2}$ \\
1 & - & - & - & 1 & - & $\mathrm{X}_{3}$ \\
$\mathbf{0}$ & - & - & - & - & 1 & $\mathbf{X}_{2}$ \\
0 & 1 & 0 & 0 & 0 & 0 & $\mathrm{X}_{4}$ \\
0 & 1 & 1 & 0 & - & - & $\mathrm{X}_{5}$ \\
- & - & 1 & 1 & - & - & $\mathrm{X}_{3}$ \\
1 & 1 & 1 & 0 & 0 & 0 & $\mathrm{X}_{6}$ \\
- & - & - & 1 & 1 & - & $\mathrm{X}_{3}$ \\
- & - & 1 & 0 & - & - & $\mathrm{X}_{2}$ \\
\hline
\end{tabular}

Table 7 . The RCOT.

\begin{tabular}{|ccccc|c|}
\hline $\mathrm{c}_{2}$ & $c_{3}$ & $c_{4}$ & $c_{5}$ & $c_{6}$ & Symbolic \\
\hline- & 1 & 0 & - & 0 & $\mathrm{X}_{1}$ \\
0 & 1 & 0 & - & 1 & $\mathrm{X}_{2}$ \\
- & 1 & 1 & 1 & - & $\mathrm{X}_{3}$ \\
0 & 1 & 0 & 0 & 0 & $\mathrm{X}_{4}$ \\
1 & 1 & 0 & - & - & $\mathrm{X}_{5}$ \\
1 & 1 & 0 & 0 & 0 & $\mathrm{X}_{6}$ \\
\hline
\end{tabular}

Consider the specification shown in Table 6. The RCOT is shown in Table 7. The CCS-1 set is $\left\{\left\{\mathrm{c}_{2}\right\},\left\{\mathrm{c}_{5}\right\},\left\{\mathrm{c}_{6}\right\}\right\}$. For calculating CCS-2 and CCS-3 we must ensure that the count of the fully specified binary strings (binary strings containing no don't cares) only need to satisfy the bounds discussed in step 3 of the algorithm in Sec. 3. Thus, CCS2 is $\left\{\left\{c_{2}, c_{5}\right\},\left\{c_{2}, c_{6}\right\}\right.$ and $\left.\left\{c_{5}, c_{6}\right\}\right\}$. CCS-3 is a null set and we choose $\left\{c_{2}, c_{5}\right\}$, a member of CCS-2, for providing the first two bits in the encoding of the symbols using 3 bits. Next, we solve the problem of assigning 0's and 1's to the don't cares in columns $\mathrm{c}_{2}$ and $\mathrm{c}_{5}$ so that the output columns representing the symbolic outputs can be merged with $c_{2}$ and $c_{5}$. We formulate the problem as a bipartite graph matching problem [2]. We form a weighted bipartite graph as follows: Set $\mathrm{V}_{1}$ contains vertices corresponding to each symbolic output with a label equal to the string formed by the entries in the row corresponding to that symbolic output under columns $c_{2}$ and $c_{5}$ of Table 7 . Set $\mathrm{V}_{2}$ contains 4 vertices each having a label that is a distinct binary pattern of length 2 . For each $u \in V_{1}$ and $v \in V_{2}$ we have an edge (u, v) of weight 1 if and only if the label of $v$ contains 1(0) in all positions in which the label of $u$ contains $1(0)$. We add two more vertices to the graph the source and the sink. There is an edge of weight 1 from the source vertex to each member of $V_{1}$. There is an edge of weight $2^{3-2}=2$ from each member of $\mathrm{V}_{2}$ to the sink.

The graph along with the source and the sink nodes and the edge weights is shown in Fig. 2. We solve the maximum network flow problem on the graph using the Ford-Fulkerson method [19]. The solid edges show the final mapping. The first two bits in the encoding of $X_{1}$, $X_{2}, X_{3}, X_{4}, X_{5}$ and $X_{6}$ are $00,01,01,10,11$ and 10 , respectively. Hence, one encoding of $X_{1}, X_{2}, X_{3}, X_{4}, X_{5}$ and $X_{6}$ is $001,010,011,100,111$ and 101 , respectively. Note that there may be multiple solutions to this bipartite graph matching problem. Hence, our algorithm can be further refined by selecting a mapping of members of $V_{1}$ to the members of $V_{2}$ based on some heuristic following the observations developed in [13] and [14].

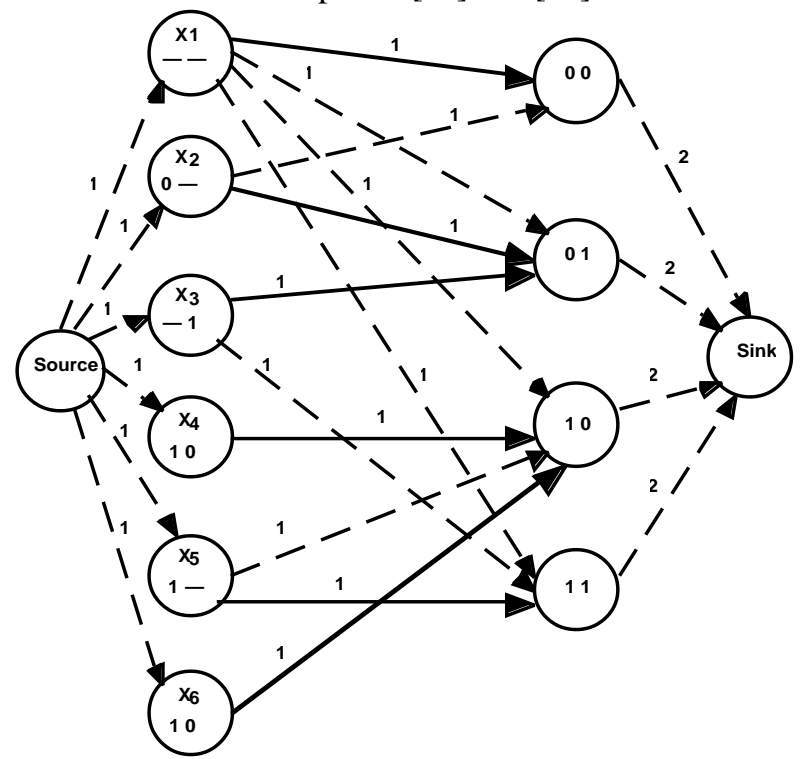

Figure 2 . The Graph for the Maximum Flow Problem.

\section{EXPERIMENTAL RESULTS}

In this section, we present experimental results. We added a symbolic output column to the MCNC FSM benchmarks and varied the number of symbols in the symbolic output column; depending on the symbol count, the number of encoding bits required were either 3 or 4 .

Table 8 . Area results for our output encoding algorithm (best case) and the worst case which never merges any output column.

\begin{tabular}{|c|c|c|c|c|}
\hline FSM & \multicolumn{2}{|c|}{3 bits for encoding } & \multicolumn{2}{c|}{ 4 bits for encoding } \\
\cline { 2 - 5 } Name & Our algo. & Worst Case & Our algo. & Worst Case \\
\hline bbara & $* 205$ & 225 & $* 285$ & 346 \\
bbsse & 316 & 364 & 316 & 378 \\
bbtas & $* 98$ & 99 & $* 103$ & 147 \\
dk14 & 223 & 266 & 223 & 294 \\
dk15 & 206 & 208 & 206 & 257 \\
dk17 & $* 182$ & 191 & $* 231$ & 304 \\
ex1 & 721 & 750 & 721 & 781 \\
ex6 & 254 & 312 & 254 & 405 \\
mc & 93 & 96 & 93 & 96 \\
opus & 235 & 260 & 235 & 356 \\
tav & 73 & 143 & 73 & 188 \\
tma & 444 & 543 & 444 & 548 \\
\hline
\end{tabular}

*: These are the cases where all the output columns could not be merged by our algorithm because the cardinality of the Bset is less than the number of bits needed to encode the symbols.

We entered symbolic values in the S-column to ensure that there exists an output encoding, for which all the columns generated due to the encoding of the symbols could be merged with the existing outputs (members of the B-set), assuming that the number of encoding bits did not exceed the number of existing non-symbolic (binary) output columns. We applied our output encoding algorithm to encode the symbolic output columns, then compacted the outputs using column compaction. We then 
optimized these specifications using sis [20]. For comparison purposes, we then encoded the symbolic outputs such that none of the outputs corresponding to the encoding bits could be merged by column compaction with the pre-existent outputs (members of the B-set). This is the worst-case encoding. The results are shown in Table 8 . Although our scheme works for both combinational and sequential logic specifications, we used FSM benchmarks because we are investigating FSM synthesis techniques for one-hot signals, as mentioned in Sec. 2 [17]. For both cases, we used NOVA [10] to encode the FSM states and used the recommended rugged script to perform multi-level logic optimization. Finally, we used the LSI Logic g10p library [21] for technology mapping. Table 8 shows the area values (in terms of LSI Logic g10p cell units) obtained using our output encoding algorithm and the worst-case output encoding where none of the encoding bits could be merged with the already existent nonsymbolic output columns by compaction.

\section{CONCLUSION}

In this paper, we have presented a new output encoding algorithm whose objective is to encode the symbols in the symbolic output column of the specification table using $\left\lceil\log _{2} n\right\rceil$ bits for $n$ symbolic outputs in such a way that the number of output functions, after performing the encoding and subsequent output column compaction, is minimum. An application of this algorithm is to generate a low-area circuit that always maintains a one-hot encoding on certain signals, even during scan or BIST operations as discussed in [17]. This algorithm can also be used as a preprocessing step for FSM state encoding. Although the algorithm produces a minimum solution and the worst case running time is exponential in the number of bits used to encode the symbolic outputs, our algorithm achieves significant speedup through iterative refinement by removing from consideration inconsistent output columns or sets of output columns. As the experimental results show, the circuits generated using our output encoding algorithm have significantly less area $(0.9 \%$ to $49 \%$ for 3 bits, $8 \%$ to $61 \%$ for 4 bits) than worst-case circuits generated without considering output column compaction during the encoding of the symbols. In this paper, we have considered specifications with a single symbolic output column. In case of multiple symbolic output columns, we can integrate our technique with the output encoding algorithm reported in [15] to achieve a minimum number of output columns in the final table.

\section{ACKNOWLEDGMENT}

This work was supported by the Advanced Research Projects Agency under prime contract No. DABT63-94-C0045. Thanks to Robert B. Norwood and Jonathan T. Y. Chang of Center for Reliable Computing, Stanford University, for their comments regarding this work.

\section{REFERENCES}

[1] Wei, R. and C Tseng, "Column Compaction and Its Application to The Control Path Synthesis," Proc. ICCAD-87, pp. 320-323, 1987.
[2] Cormen, T. H., et. al., Introduction to Algorithms, The MIT Press and McGraw-Hill, 1989.

[3] Brayton, R. K., et. al., Logic Minimization Algorithms for VLSI Synthesis, Kluwer Publishers, 1984.

[4] Ashar, P., S. Devadas and A. R. Newton, Sequential Logic Synthesis, Kluwer Academic Publishers, 1991.

[5] Dolotta, T. A., and E. J. McCluskey, "The Coding of Internal States of Sequential Circuits," IEEE Trans. Comput., EC-13, pp. 549-562, Oct. 1964.

[6] De Micheli, G., et. al., "Optimal State Assignment for Finite State Machines," IEEE Trans. on CAD., CAD 4(3), 269285, July 1985.

[7] Du, X., et. al. "MUSE: A MUltilevel Symbolic Encoding Algorithm for State Assignment," IEEE Trans. on CAD, 10(1), pp. 28-38, Jan. 1991.

[8] Lin, B. and A. R. Newton, "Synthesis of Multiple Level Logic from Symbolic High-Level Description Languages," VLSI 89, pp. 187-196, Elsevier, 1990.

[9] Tumbush, G. L. and J. E. Brandeberry, "A State Assignment Technique for Sequential Machines using J-K Flip-Flops," IEEE Trans. Comput., pp. 85-86, Jan. 1974.

[10] Villa, T. and A. Sangiovanni-Vincentelli, "NOVA: State Assignment of Finite State Machines for Optimal Two-Level Logic Implementation", IEEE Trans. on CAD, 9(9), pp. 905924, Sept. 1990.

[11] Buijs, F., et. al., "Synthesis of Multi-Level Logic with one symbolic input," EDAC-91, pp. 60-64, 1991.

[12] Yang, S., et. al., "Optimum and Sub-optimum Algorithms for Input Encoding and Its Relation to Logic Minimization," IEEE Trans. on CAD, 10(1), pp. 4-12, Jan. 1991.

[13] Devadas, S. and A. R. Newton, "Exact Algorithms for Output Encoding, State Assignment and Four Level boolean Minimization," IEEE Trans. on CAD, 10(1), pp. 13-27, Jan. 1991.

[14] Saldanha, A. and R. H. Katz, "PLA Optimization Using Output Encoding," ICCAD, pp. 478-481, 1988.

[15] Binger, D. and D. W. Knapp, "Encoding Multiple Outputs for Improved Column Compaction," Proc. ICCAD-91, pp. 230-233, 1991.

[16] McCluskey, E. J., Logic Design Principles with Emphasis on Testable Semicustom circuits, Prentice-Hall, Eaglewood Cliffs, NJ, USA, 1986.

[17] Mitra, S., L. J. Avra and E. J. McCluskey, "Scan Synthesis for One-hot Signals", Proc. ITC, Nov. 1997.

[18] Mitra, S., L. J. Avra and E. J. McCluskey, "An Output Encoding Problem and a Solution Technique", Technical Report, Center for Reliable Computing, Stanford University, CRC TR 97-1, 1997.

[19] Ford, L. R. and D. R. Fulkerson, Flows in Networks, Princeton University Press, 1962.

[20] Sentovich, E., et. al., "SIS: A System for Sequential Circuit Synthesis", ERL Memo. No. UCB/ERL M92/41, Department of EECS, UC Berkeley, CA 94720.

[21] G10-p Cell-Based ASIC Products Databook, LSI Logic, May 1996. 